\title{
The impact of ethnic minority status on tuberculosis diagnosis and treatment delays in Hunan Province, China
}

Beth Gilmour ${ }^{1 *}$, Zuhui Xu ${ }^{3,4+}$, Liqiong Bai ${ }^{4}$, Kefyalew Addis Alene ${ }^{1,2}$ and Archie C. A. Clements ${ }^{1,2}$

\begin{abstract}
Background: Tuberculosis (TB) continues to be a major public health challenge in China. Understanding TB management delays within the context of China's unique ethnic diversity may be of value in tackling the disease. This study sought to evaluate the impact of ethnic minority status on TB diagnosis and treatment delays.

Methods: This retrospective cohort study was conducted on patients diagnosed with TB in Hunan Province, China between 2013 and 2018. Diagnosis delay was defined as the time interval between the onset of symptoms and the date of diagnosis. Treatment delay was defined as the time interval between diagnosis and treatment commencement. Univariable and multivariable logistic regression models were used to identify factors associated with TB diagnosis and treatment delay, including ethnic minority status. Adjusted odds ratios (AOR) with $95 \%$ confidence intervals (Cl) were calculated to assess the strength of association between the dependant and independent variables.
\end{abstract}

Results: A total of 318,792 TB patients were included in the study with a mean age of 51.7 years (SD 17.7). The majority of patients were male (72.6\%) and Han ethnicity (90.6\%). The odds of experiencing diagnosis delay (>21 days) were significantly higher for Tujia (AOR: 1.46, 95\% Cl: 1.41, 1.51), Miao (AOR: 1.31, 95\% Cl: 1.26, 1.37), Dong (AOR: 1.97, 95\% $\mathrm{Cl}: 1.85,2.11$ ), Yao (AOR: 1.27, 95\% Cl: 1.17, 1.37), and Bai (AOR: 1.45, 95\% Cl: 1.22, 1.74) ethnic minorities compared to the Han majority. The odds of experiencing treatment delay (> 15 days) were significantly lower for five of the seven ethnic minority groups relative to the Han majority: Tujia (AOR 0.92, 95\% Cl 0.88, 0.96), Miao (AOR 0.74, 95\% Cl 0.70, 0.79), Dong (AOR 0.87, 95\% Cl 0.81, 0.95), Yao (AOR 0.20, 95\% Cl 0.17, 0.24) and 'other' (ethnic minorities that individually represented $<0.1 \%$ of the patient population) (AOR 0.70, $955 \mathrm{Cl} 0.51,0.97$ ).

Conclusions: This study shows ethnic minority status to be a significant risk factor in diagnosis delay, but for it to reduce the odds of treatment delay. Further research is required to determine the underlying causes of diagnosis delay within ethnic minority populations.

Keywords: Tuberculosis, Ethnic minority, Diagnosis delay, Treatment delay, China

*Correspondence: beth.gilmour@postgrad.curtin.edu.au

${ }^{\dagger}$ Beth Gilmour and Zuhui Xu equally contributed to this work

${ }^{1}$ Faculty of Health Sciences, Curtin University, Western Australia, Kent St,

Bentley, WA 6102, Australia

Full list of author information is available at the end of the article

\begin{abstract}
Background
Tuberculosis (TB) is currently second to Coronavirus Disease 2019 (COVID -19) as a leading cause of death from a single infectious agent [1], claiming a life every $22 \mathrm{~s}$ [2]. Prior to the COVID-19 pandemic, TB was the leading cause of death [3] and throughout history is thought to have claimed more lives than any other microorganism [4]. TB is caused by Mycobacterium
\end{abstract}


tuberculosis (MTB), an airborne pathogen that most commonly affects the lungs (pulmonary TB) albeit the pathogen can affect all organs (extrapulmonary TB). Although the disease can be cured, escalating drug resistance presents a global health security threat [3].

In 2019, China ranked third for the greatest number of new TB cases globally [3] with 833,000 people falling ill to the disease [5]. To address the burden of disease, China is in the process of comprehensive public health system reforms, including setting the goal of universal health coverage and transformation of the TB service delivery model [6-8]. In 1991, China launched its National Tuberculosis Control Programme (NTP) based on the World Health Organization (WHO) recommended Directly Observed Treatment Short-course (DOTS) strategy. The NTP aims to provide TB diagnosis and treatment services free of charge, with a focus on the poor, ethnic minorities and other vulnerable population groups. $[9,10]$.

Fundamental to the success of national TB control programs, is early detection and prompt and appropriate treatment [11]. Delays in timely diagnosis and treatment lead to disease progression, poor treatment outcomes, increased risk of transmission and an exacerbation of the socioeconomic consequences of the disease. [12].

A systematic review and meta-analysis of patient and diagnosis delays in China, found an array of contributing factors [13]. Factors included indicators of low socio-economic status (e.g., low level of education, low disposable income, lack of health insurance); rural residence; female sex; initial consultation with traditional healers and resource constraints within the health care service [13]. However, to our knowledge, the impact of ethnic minority status upon diagnosis and treatment delays within China's TB patient population has not been investigated.

China has a unique socio-cultural environment, and understanding this within the context of delays in TB management could be of value in combating the disease [13]. Hunan Province, located in south-central China, is one of the most populous divisions of the country where ethnic minority groups represent 10.1 percent of the population [14]. Despite significant investments in TB control and treatment strategies by the Hunan government [14], which have reduced the burden of disease [15], Hunan remains a high TB burden province. [16, 17].

This study aimed to evaluate the impact of ethnic minority status on the time to diagnosis and the time to treatment among patients registered in Hunan Province between 2013 and 2018.

\section{Methods}

\section{Study design and data sources}

Operating under the provincial health committee, the Hunan Tuberculosis Control Institute is responsible for the province's TB control and prevention, and research and development [18]. This is a retrospective cohort study conducted on patients diagnosed with pulmonary and extrapulmonary TB in Hunan Province between 2013 and 2018 inclusive. Data were obtained from the internet-based TB management system administered by the TB Control Institute of Hunan Province (TBCIHP).

The date of symptom onset and the date of any previous diagnosis (if any) were recorded in the system on the basis of information provided by the patient. The date of TB diagnosis and date of treatment commencement were recorded by health professionals at the designated TB institutions. Demographic data e.g., ethnic group, sex, age, occupation, year of registration at the designated TB institution and residential address were also available.

\section{Definitions}

Total delay is defined as the timeframe between the onset of disease and the start of treatment [19]. The total delay can be classified in two ways- as the sum of the diagnosis delay (time between the onset of symptoms and diagnosis) and the treatment delay (time between diagnosis and treatment commencement) or as the patient delay (time between onset of symptoms and consultation with a health care provider) and the health system delay (time between patient consultation and start of treatment, Fig. 1) [19]. This study evaluated diagnostic and treatment delays.

Hunan's TB institutions follow a TB diagnosis based on WHO recommended methods, e.g., clinical assessment based on symptoms, sputum smear microscopy, chest $\mathrm{x}$-ray, sputum culture and molecular detection [20].

China's ethnic classification system recognizes 55 minority groups in addition to the Han majority [21]. For this study, associations between diagnosis delay and treatment delay with Tujia, Miao, Dong, Yao, Bai, Mongolian and 'other' ethnic minority group status were estimated relative to the Han majority. The 'other' ethnic minority group comprised the summation of all other ethnic minority groups, who constitute $<0.1 \%$ of the patient population. The 'other' group included Buyi, Dai, Gelao, Hani, Hui, Jingpo, Kazakh, Kirgiz, Korean, Lahu, Li, Lisu, Manchu, Salar, She, Tibetan, Tu, Uighur, Wa, Yao, Yi, and Zhuang ethnic minorities.

Definitions pertaining to the other clinical descriptors/ variables analyzed are detailed in Table 1. 


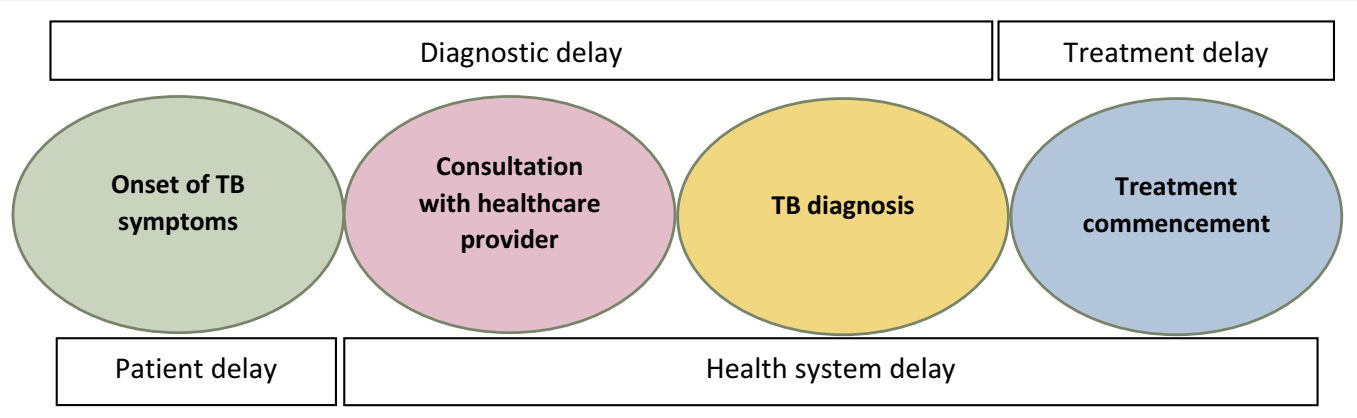

Total delay

Fig. 1 Conceptual framework for TB diagnosis and treatment delay [19]. Total delay= time between the onset of symptoms and the commencement of treatment. Diagnosis delay = time between onset of symptoms and diagnosis. Treatment delay=time between diagnosis and treatment commencement. Patient delay = time between onset of symptoms and consultation with healthcare provider. Health care system delay $=$ time between consultation and treatment commencement

\section{Statistical analysis}

Data were translated from Mandarin to English, checked for completeness, cleaned, and entered into STATA version 16.1 (StataCorp, College Station, TX) for analysis. Frequency and cross-tabulation were used to cross check data completeness.

Descriptive statistics were used to summarize data and define characteristics of the patient population. Treatment and diagnosis delays were calculated in days and summarized, using the median and interquartile range (IQR) because the data showed a non-normal distribution.

To dichotomize data, the median (21 days) was used to define diagnosis delay and the upper quartile (15 days) was used to define treatment delay. Categorical variables were described by counts and percentages, and continuous and normally distributed variables were summarized by means and standard deviations (SD). Univariable logistic regression models were fitted and Crude Odds Ratios (COR) with 95\% Confidence Intervals (CI) reported. Multicollinearity between independent variables was assessed using variance inflation factors (VIF) and variables with VIF $>5$ were excluded from the final multivariable analysis.

All variables assessed in the univariable models were fitted into multivariable logistic regression models. Adjusted odds ratios and 95\% confidence intervals (CI) were computed to measure the association between the dependent (i.e., diagnosis and treatment delays) and independent variables (i.e., ethnic minority status, sex, occupation year of patient registration, residential address, patient enrolment classification, diagnosis institution and whether a patient was severely ill). Variables with a $p$-value $<0.05$ in the multivariable analysis were considered as having a statistically significant association with the outcome (diagnosis or treatment delay). Additional models were created for sensitivity analyses to evaluate a 14 day delay used by some studies [13, 22], compared a 21 day diagnosis delay used by others [23-27]. An analysis was also conducted using the median ( $>1$ day) to define treatment delay.

To evaluate the outcome variables (i.e., diagnosis and treatment delay) in their continuous form sensitivity analyses were undertaken using a negative binomial regression model.

An additional model was constructed to determine treatment delay for patients with two TB diagnosis dates. 'New patient' treatment delay was defined as the time period (days) between the date of the second diagnosis and treatment commencement. This analysis was undertaken to mitigate the risk of treatment being administered between a patients first diagnosis and a subsequent diagnosis, a timeframe for which we had no data.

\section{Ethics statement}

Ethical clearance was obtained from Curtin University (HRE2019-0581) and written permission to access the data granted from TBCIHP. Medical records of the patient population were de-identified to preserve privacy. Because this study used secondary, de-identified data, informed patient consent was not required.

\section{Results \\ Socio-demographic and clinical characteristics of the patients}

A total of 318,792 TB patients registered in Hunan Province between 2013 and 2018 were included in this study. The sociodemographic characteristics of the patients are presented in Table 2. The majority of patients were male $(72.6 \%)$ and the study population 
Table 1 Definitions of clinical variables included in the study

\begin{tabular}{|c|c|}
\hline Variable/Demographic descriptor & Definition \\
\hline \multicolumn{2}{|l|}{ Residential address } \\
\hline Local & Patients who reside in local counties \\
\hline Intra-provincial & Patients who reside in other counties within the province \\
\hline Inter-provincial & Patients who reside in provinces other than Hunan \\
\hline Foreign nationality & Patients who reside in other countries \\
\hline \multicolumn{2}{|l|}{ Patient enrolment classification } \\
\hline Consultation due to symptoms & Patients who consult the TB institution due to symptoms \\
\hline Referral & Patients who are referred to the TB institution due to symptoms \\
\hline Contact tracing & TB patients identified by contact tracing \\
\hline Health check & TB patients who are identified as a result of a health check \\
\hline \multicolumn{2}{|l|}{ TB diagnosis results } \\
\hline Etiological examine negative & TB cases identified on the basis of symptoms \\
\hline Smear positive & Positive Acid-Fast Bacillus test \\
\hline Extrapulmonary TB & TB identified in organs other than the lungs \\
\hline Culture positive & TB positive sputum culture \\
\hline Severely ill & Patients with miliary TB, cavities, TB empyema or serious damage to one or more organ caused by TB disease \\
\hline \multicolumn{2}{|l|}{ Drug resistance pattern } \\
\hline Drug susceptible TB & M.tuberculosis that is susceptible to first line antibiotics (isoniazid, rifampin, ethambutol, and pyrazinamide) \\
\hline MDR-TB & M.tuberculosis that is resistant to isoniazid and rifampicin \\
\hline Mono-resistant TB & M.tuberculosis resistant to a single first line antibiotic \\
\hline \multicolumn{2}{|l|}{ Diagnosis institution } \\
\hline CDC & Centre for disease control and prevention that has a TB clinic \\
\hline Hospital & General hospital \\
\hline TB dispensary & Specialized TB hospital (TB patients only) \\
\hline Other & Other health institution or hospital not covered by above classifications \\
\hline \multicolumn{2}{|l|}{ Registration category } \\
\hline New patient & $\begin{array}{l}\text { TB patients who have never taken anti-TB drugs, or who have been receiving irregular treatment for less than one } \\
\text { month }\end{array}$ \\
\hline Relapse & $\begin{array}{l}\text { TB patients with a history of disease, who complete a full course of chemotherapy and appear cured according to } \\
\text { symptoms, but who return a smear positive sputum sample }\end{array}$ \\
\hline Return after default & $\begin{array}{l}\text { TB patients who receive chemotherapy for } \geq 1 \text { month but discontinue therapy for } \geq 2 \text { months and then return for } \\
\text { treatment }\end{array}$ \\
\hline Initial treatment failed & $\begin{array}{l}\text { New sputum smear positive TB patients with positive sputum smear microscopy results at the end of the } 5 \text { th } \\
\text { month or after completion of therapy; and sputum smear negative TB patients with a positive smear result for } \\
\text { any sputum sample }\end{array}$ \\
\hline Chronic patient & Positive sputum examination results after several episodes of irregular therapy \\
\hline \multicolumn{2}{|l|}{ Treatment category } \\
\hline Initial treatment & TB patients who have never taken anti-TB drugs \\
\hline Retreatment & Patient who has history of TB treatment \\
\hline \multicolumn{2}{|l|}{ TB treatment } \\
\hline Accept treatment & Patient who accepts the recommended treatment regime \\
\hline Reject treatment & Patient who rejects the recommended treatment regime \\
\hline
\end{tabular}

had a mean age of 51.75 years (SD 17.67). Patients of Han ethnicity formed the majority (90.6\%) with the remainder of the patient population represented by 28 ethnic minority groups (ethnicity data were not available for 4 patients). Seventy-eight percent of patients were employed in the agricultural sector. Most patients were new (95.7\%), with the majority not severely ill
(96.2\%) and in receipt of a drug susceptible TB diagnosis $(87.2 \%)$.

\section{Median time to diagnosis and treatment by ethnic minority status}

Table 3 illustrates median time to diagnosis and treatment by study characteristics. Across all patients, the 
Table 2 Sociodemographic and clinical characteristics of TB patients registered in Hunan Province, China, 2013-2018

\begin{tabular}{|c|c|c|}
\hline Variable & Number & Percent \\
\hline \multicolumn{3}{|l|}{ Sex } \\
\hline Male & 231,495 & 72.62 \\
\hline Female & 87,297 & 27.38 \\
\hline \multicolumn{3}{|l|}{ Age (years) } \\
\hline \multicolumn{3}{|l|}{ Mean $=51.75 ;$ SD 17.67} \\
\hline $0-10$ & 346 & 0.11 \\
\hline $11-20$ & 15,767 & 4.95 \\
\hline $21-30$ & 37,135 & 11.65 \\
\hline $31-40$ & 30,470 & 9.56 \\
\hline $41-50$ & 56,269 & 17.65 \\
\hline $51-60$ & 62,606 & 19.64 \\
\hline $61-70$ & 69,209 & 21.71 \\
\hline $71-80$ & 38,807 & 12.17 \\
\hline $81-101$ & 8183 & 2.57 \\
\hline \multicolumn{3}{|l|}{ Occupation } \\
\hline Agriculture ${ }^{\sim}$ & 249,093 & 78.14 \\
\hline Housekeeping ${ }^{\$}$ & 30,802 & 9.66 \\
\hline Education $^{\Delta}$ & 10,679 & 3.35 \\
\hline Commercial services/civil servant & 7818 & 2.45 \\
\hline Migrant worker & 2601 & 0.82 \\
\hline Healthcare & 1009 & 0.32 \\
\hline Hospitality & 612 & 0.19 \\
\hline Other & 16,178 & 5.07 \\
\hline \multicolumn{3}{|l|}{ Ethnicity } \\
\hline Han & 288,802 & 90.59 \\
\hline Tujia & 13,680 & 4.29 \\
\hline Miao & 8460 & 2.65 \\
\hline Dong & 4033 & 1.27 \\
\hline Yao & 2662 & 0.84 \\
\hline Bai & 509 & 0.16 \\
\hline Mongolian & 349 & 0.11 \\
\hline Other* & 293 & 0.09 \\
\hline \multicolumn{3}{|l|}{ Residential address } \\
\hline Local & 310,343 & 97.35 \\
\hline Intra-provincial & 6215 & 1.95 \\
\hline Inter-provincial & 2182 & 0.68 \\
\hline Foreign nationality & 52 & 0.02 \\
\hline \multicolumn{3}{|l|}{ Patient enrolment classification } \\
\hline Consultation due to symptoms & 117,834 & 36.96 \\
\hline Referral & 103,261 & 32.39 \\
\hline Contact tracing & 93,183 & 29.23 \\
\hline Health check & 3179 & 1.00 \\
\hline Other & 1335 & 0.42 \\
\hline \multicolumn{3}{|l|}{ TB diagnosis results } \\
\hline Etiological examination negative & 189,129 & 59.32 \\
\hline Smear positive & 122,006 & 38.27 \\
\hline Extrapulmonary TB & 5609 & 1.76 \\
\hline Culture positive & 1355 & 0.43 \\
\hline Molecular diagnosis positive & 693 & 0.22 \\
\hline
\end{tabular}

Table 2 (continued)

\begin{tabular}{|c|c|c|}
\hline Variable & Number & Percent \\
\hline \multicolumn{3}{|l|}{ Severely ill } \\
\hline No & 306,534 & 96.15 \\
\hline Yes & 12,258 & 3.85 \\
\hline \multicolumn{3}{|l|}{ Drug resistance pattern } \\
\hline Drug susceptible TB & 15,555 & 87.23 \\
\hline MDR-TB & 1248 & 7.00 \\
\hline Mono-resistant TB & 1030 & 5.78 \\
\hline \multicolumn{3}{|l|}{ Diagnosis institution } \\
\hline Centre for Disease Control \& Prevention (CDC) & 278,707 & 88.15 \\
\hline Hospital & 33,104 & 10.47 \\
\hline TB dispensary & 4276 & 1.35 \\
\hline Other & 69 & 0.02 \\
\hline \multicolumn{3}{|l|}{ Registration category } \\
\hline New patient & 305,218 & 95.74 \\
\hline Relapse & 12,179 & 3.82 \\
\hline Return after default & 350 & 0.11 \\
\hline Initial treatment failed & 279 & 0.09 \\
\hline Chronic patient & 122 & 0.04 \\
\hline Other & 644 & 0.20 \\
\hline \multicolumn{3}{|l|}{ Treatment category } \\
\hline Initial treatment & 305,306 & 95.77 \\
\hline Retreatment & 13,486 & 4.23 \\
\hline \multicolumn{3}{|l|}{ TB treatment } \\
\hline Accept treatment & 318,324 & 99.86 \\
\hline Reject treatment & 462 & 0.17 \\
\hline
\end{tabular}

$\sim$ Agriculture includes famer, herdsman, fisherman

\$ Housekeeping includes housekeeping, childcare, retired and unemployed

${ }^{\Delta}$ Education includes students and teachers

* Other are represented by: Buyi, Dai, Gelao, Hani, Hui, Jingpo, Kazakh, Kirgiz, Korean, Lahu, Li, Lisu, Manchu, Salar, She, Tibetan, Tu, Uighur, Wa, Yao, Yi, and Zhuang ethnic groups

median time to diagnosis was 21 days (IQR 7-50 days), and the median time to treatment was 1 day (IQR $0-15$ days).

Results show differences in median time to diagnosis and treatment across different ethnic groups. The median time to diagnosis for the Han majority population was 20 days (IQR 6-49 days); 30 days (IQR 7-65 days) for Tujia; 27 days (IQR 10-61 days) for Miao; 35 days (IQR 10-75 days) for Dong; 24 days (IQR 7-58 days) for Yao; 28 days (IQR 7-51 days) for Bai; 23 days (IQR 7-52 days) for Mongolian and 16 days (IQR 3-46 days) for 'other' ethnic minority groups. For each of the ethnic groups, the median time to diagnosis by year of patient registration is represented graphically in Fig. 1 of the Additional file 1 . The median time to treatment was 1 day (IQR 0-16 days) for Han; 1 day (IQR 0-9 days) for Tujia; 1 day (IQR 0-9 days) for Miao; 1 day (IQR 0-9 days) for Dong; 0 days (IQR 0-2 days) for Yao; 2 days (IQR 0-9 days) for 
Table 3 Median time from symptom onset to diagnosis and from diagnosis to treatment commencement for TB patients registered in Hunan Province, China 2013-2018, by demographic and clinical characteristics

\begin{tabular}{|c|c|c|c|}
\hline & Number of patients (\%) & $\begin{array}{l}\text { Median time to diagnosis } \\
\text { (days) }\end{array}$ & $\begin{array}{l}\text { Median time } \\
\text { to treatment } \\
\text { (days) }\end{array}$ \\
\hline All patients & 318,792 & $21(\mathrm{IQR} 7-50)$ & 1 (IQR 0-15) \\
\hline \multicolumn{4}{|l|}{ Ethnicity } \\
\hline Han & $288,802(90.59)$ & 20 (IQR 6-49) & 1 (IQR 0-16) \\
\hline Tujia & $13,680(4.29)$ & $30($ IQR 7- 65) & 1 (IQR 0-9) \\
\hline Miao & $8460(2.65)$ & 27 (IQR 10-61) & 1 (IQR 0-9) \\
\hline Dong & $4033(1.27)$ & 35 (IQR 10-75) & 1 (IQR 0-9) \\
\hline Yao & $2662(0.84)$ & $24(\mathrm{IQR} 7-58)$ & $0(\mathrm{IQR} 0-2)$ \\
\hline Bai & $509(0.16)$ & 28 (IQR 7-51) & 2 (IQR 0-9) \\
\hline Mongolian & $349(0.11)$ & 23 (IQR 7-52) & $1(\mathrm{IQR} 0-12)$ \\
\hline Other* & $293(0.09)$ & 16 (IQR 3-46) & 1 (IQR 0-9) \\
\hline \multicolumn{4}{|l|}{ Sex } \\
\hline Male & $231,495(72.62)$ & $21(I Q R$ 7-50) & 1 (IQR 0-14) \\
\hline Female & $87,297(27.38)$ & 21 (IQR 7-51) & $1($ IQR 0-17) \\
\hline \multicolumn{4}{|l|}{ Age } \\
\hline$<18$ years & $7155(2.24)$ & 14 (IQR 3-36) & 2 (IQR 0-17) \\
\hline$\geq 18$ years & $311,637(97.76)$ & 21 (IQR 7-51) & 1 (IQR 0-15) \\
\hline \multicolumn{4}{|l|}{ Occupation } \\
\hline Agriculture ${ }^{\sim}$ & $249,093(78.14)$ & $22(\mathrm{IQR} 7-54)$ & $1(\mathrm{IQR} 0-12)$ \\
\hline Housekeeping ${ }^{\$}$ & $30,802(9.66)$ & 19 (IQR 5-46) & 4 (IQR 0-27) \\
\hline Education $^{\Delta}$ & $10,679(3.35)$ & 13 (IQR 3-33) & $2(\mathrm{IQR} 0-20)$ \\
\hline Commercial services/civil servant & $7818(2.45)$ & 16 (IQR 4-39) & $7(\mathrm{IQR} 0-30)$ \\
\hline Migrant worker & $2601(0.82)$ & 19 (IQR 7-46) & $1(\mathrm{IQR} 0-10)$ \\
\hline Healthcare & $1009(0.32)$ & 15 (IQR 4-36) & 4 (IQR 0-25) \\
\hline Hospitality & $612(0.19)$ & 14 (IQR 3-32) & 6 (IRQ 0-27) \\
\hline Other & $16,178(5.07)$ & 16 (IQR 4-40) & 2 (IQR 0-23) \\
\hline \multicolumn{4}{|l|}{ Year } \\
\hline 2013 & $56,198(17.63)$ & 21 (IQR 6-55) & $1(\mathrm{IRQ} 0-14)$ \\
\hline 2014 & $55,815(17.51)$ & $21(\mathrm{IQR} 7-51)$ & 1 (IQR 0-14) \\
\hline 2015 & $55,196(17.31)$ & $21($ QQR 7-50) & $1($ IQR 0-14) \\
\hline 2016 & $49,996(15.68)$ & $22(I Q R$ 7-52) & 1 (IQR 0-13) \\
\hline 2017 & $49,843(15.63)$ & 21 (IQR 6-48) & $1($ IQR 0-16) \\
\hline 2018 & $51,744(16.23)$ & 19 (IQR 6-48) & 1 (IQR 0-18) \\
\hline \multicolumn{4}{|l|}{ Residential address } \\
\hline Local & $310,343(97.35)$ & $21(I Q R$ 7-50) & 1 (IQR 0-14) \\
\hline Intra-provincial (within province) & $6215(1.95)$ & 27 (IQR 6-59) & $2($ IQR 0-30) \\
\hline Inter-provincial (between provinces) & $2182(0.68)$ & 18 (IQR 4-44) & 5 (IQR 0-28) \\
\hline Foreign nationality & $52(0.02)$ & $29.5($ IQR 7.5-65) & $0($ (IQR 0-4) \\
\hline \multicolumn{4}{|l|}{ Patient enrolment classification } \\
\hline Consultation due to symptoms & $117,834(36.96)$ & $26(\mathrm{IQR} 11-60)$ & $0(\mathrm{IQR} 0-1)$ \\
\hline Referral & $103,261(32.39)$ & 17 (IQR 5-45) & $1(\mathrm{IQR} 0-12)$ \\
\hline Contact tracing & $93,183(29.23)$ & 19 (IQR 4-48) & 17 (IQR 0-38) \\
\hline Health check & $3179(1.00)$ & $3(\mathrm{IQR} 0-14)$ & 0 (IQR 0-3) \\
\hline Other & $1335(0.42)$ & 15 (IQR 4-34) & 8 (IQR 1-28) \\
\hline \multicolumn{4}{|l|}{ Diagnosis institution } \\
\hline CDC & $278,707(88.15)$ & $21(I Q R$ 7-51) & 1 (IQR 0-15) \\
\hline Hospital & $33,104(10.47)$ & 20 (IQR 6-45) & 0 (IQR 0-9) \\
\hline TB dispensary & $4276(1.35)$ & 19 (IQR 10-35) & $11(\mathrm{IQR} 2-18)$ \\
\hline
\end{tabular}


Table 3 (continued)

\begin{tabular}{|c|c|c|c|}
\hline & Number of patients (\%) & $\begin{array}{l}\text { Median time to diagnosis } \\
\text { (days) }\end{array}$ & $\begin{array}{l}\text { Median time } \\
\text { to treatment } \\
\text { (days) }\end{array}$ \\
\hline Other & $69(0.02)$ & $16(\mathrm{IQR} 5-38)$ & $1(\mathrm{QQR} 0-7)$ \\
\hline \multicolumn{4}{|l|}{ Severely ill } \\
\hline No & $306,534(96.15)$ & $21(\mathrm{IQR} 7-50)$ & $1(\mathrm{IQR} 0-15)$ \\
\hline Yes & $12,258(3.85)$ & 28 (IQR 9-62) & $1($ IQR 0-13) \\
\hline \multicolumn{4}{|c|}{ Agriculture includes famer, herdsman, fisherman } \\
\hline \multicolumn{4}{|c|}{${ }^{\$}$ Housekeeping includes housekeeping, childcare, retired and unemployed } \\
\hline \multicolumn{4}{|c|}{${ }^{\Delta}$ Education includes students and teachers } \\
\hline
\end{tabular}

Bai; 1 day (IQR 0-12 days) for Mongolian and 1 day (IQR 0-9 days) for 'other' ethnic minority groups.

The median time to diagnosis (21 days) was used to define delay in subsequent analyses. For clinical relevance, the upper quartile (15 days) was used to define treatment delay, with a sensitivity analysis conducted at the median (1 day).

\section{Factors associated with tuberculosis diagnosis delays}

Results of univariable and multivariable logistic regression models to identify factors associated with diagnosis delay are detailed in Table 4. Univariable analysis shows five of the seven ethnic minority groups (i.e., Tujia, Miao, Dong, Yao, and Bai) to have significantly longer diagnosis delays than the reference Han majority. The same five ethnic minority groups had significant greater odds of experiencing diagnosis delays in the multivariable models. The odds of experiencing diagnosis delays relative to the Han majority were significantly higher for Tujia (adjusted odds ratio (AOR): 1.46, 95\% CI: 1.41, 1.51), Miao (AOR: 1.31, 95\% CI: 1.26, 1.37), Dong (AOR: 1.97, 95\% CI: 1.85, 2.11), Yao (AOR: 1.27, 95\% CI: 1.17, 1.37), and Bai (AOR: 1.45, 95\% CI: 1.22, 1.74) ethnic minorities. Differences in diagnosis delay for the Mongolian ethnic group (AOR 1.20, 95\% CI 0.97, 1.48) and the 'other' ethnic minorities (AOR $0.9295 \%$ CI $0.73,1.17$ ) relative to the Han majority were not significant.

The results of the sensitivity analysis using $>14$ days to define a diagnosis delay are presented in the Additional file (Additional file 1: Table S1). The analysis shows there to be no difference (14 day vs. 21 day) in the ethnic minority groups that are associated with a significant diagnosis delay relative to the Han majority.

Other variables found to be associated with a $>21$ day diagnosis delay in the multivariable analysis include female sex (AOR: 1.04; 95\% CI 1.03,1.06); increasing age (AOR 1.004 per one year increase; 95\% CI 1.003, 1.004); agriculture (AOR 1.25; 95\% CI 1.19, 1.31) and housekeeping (AOR 1.17; 95\% CI 1.11, 1.23) occupations relative to the commercial services/civil servants; patient registrations in 2016 (AOR 1.06, 95\% CI 1.03, 1.09) relative to 2013; residing within the province (AOR 1.48; 95\% CI 1.41, 1.56) relative to being local; and being severely ill (AOR 1.35; 95\% CI 1.31, 1.41).

The negative binomial regression assessment of factors associated with time to diagnosis is detailed in the Additional file (Additional file 1: Table S2).

\section{Factors associated with tuberculosis treatment delays}

Results of univariable and multivariable regression models to identify factors associated with treatment delay $>15$ days are detailed in Table 5 . The multivariable analysis shows that five of the seven ethnic minority groups have significantly lower odds of treatment delay than the Han majority: Tujia (AOR 0.92, 95\% CI 0.88, 0.96), Miao (AOR 0.74, 95\% CI 0.70, 0.79), Dong (AOR $0.87,95 \%$ CI $0.81,0.95$ ), Yao (AOR $0.20,95 \%$ CI 0.17 , 0.24 ) and 'other' (AOR 0.70, 95\% CI 0.51, 0.97).

A sensitivity analysis using the median ( $>1$ day) to define treatment delay is presented in the Additional file (Additional file 1: Table S3). This analysis shows a variety of treatment delays across the different ethnic groups, with no clear trend detectable.

The other variables associated with $>15$ day treatment delay in the multivariable model include female sex (AOR 1.07; 95\% CI 1.05, 1.09); increasing age (AOR 1.001 per one year increase; 95\% CI 1.0004, 1.002); 2018 as the year of registration relative to 2013 (AOR 1.08; 95\% CI $1.05,1.12)$; residing inter-provincially relative to being local (AOR 1.14; 95\% CI 1.04, 1.26); being enrolled due to referral (AOR 3.62, 95\% CI 3.52, 3.72), contact tracing (AOR 14.45, 95\% CI 14.06, 14.84) and for other reasons (AOR 7.78, 95\% CI 6.94, 8.72) relative to consultation due to symptoms and being diagnosed at a TB dispensary (AOR 3.32; 95\% CI 3.09, 3.56) relative to a CDC. 
Table 4 Univariable and multivariable regression assessment of factors associated with 21 day diagnosis delay in TB patients registered in Hunan Province, 2013-2018

\begin{tabular}{|c|c|c|c|c|c|}
\hline & Number of patients (\%) & $\begin{array}{l}\text { Univariable odds } \\
\text { ratio }(95 \% \mathrm{Cl})\end{array}$ & $\begin{array}{l}\text { Univariable } p \\
\text { value }\end{array}$ & $\begin{array}{l}\text { Multivariable odds } \\
\text { ratio }(95 \% \mathrm{CI})\end{array}$ & $\begin{array}{l}\text { Multivariable } \\
p \text { value }\end{array}$ \\
\hline \multicolumn{6}{|l|}{ Ethnicity } \\
\hline Han & $288,802(90.59)$ & 1.00 & & 1.00 & \\
\hline Tujia & $13,680(4.29)$ & $1.38(1.33,1.43)$ & 0.000 & $1.46(1.41,1.51)$ & 0.000 \\
\hline Miao & $8460(2.65)$ & $1.29(1.77,2.02)$ & 0.000 & $1.31(1.26,1.37)$ & 0.000 \\
\hline Dong & $4033(1.27)$ & $1.89(1.77,2.02)$ & 0.000 & $1.97(1.85,2.11)$ & 0.000 \\
\hline Yao & $2662(0.84)$ & $1.18(1.10,1.28)$ & 0.000 & $1.27(1.17,1.37)$ & 0.000 \\
\hline Bai & $509(0.16)$ & $1.30(1.09,1.55)$ & 0.004 & $1.45(1.22,1.74)$ & 0.000 \\
\hline Mongolian & $349(0.11)$ & $1.21(0.98,1.49)$ & 0.078 & $1.20(0.97,1.48)$ & 0.099 \\
\hline Other* & $293(0.09)$ & $0.81(0.64,1.02)$ & 0.067 & $0.92(0.73,1.17)$ & 0.494 \\
\hline \multicolumn{6}{|l|}{ Sex } \\
\hline Male & $231,495(72.62)$ & 1.00 & & 1.00 & \\
\hline Female & $87,297(27.38)$ & $1.02(1.00,1.03)$ & 0.021 & $1.04(1.03,1.06)$ & 0.000 \\
\hline Age & $318,792(100)$ & $1.01(1.01,1.01)$ & 0.000 & $1.004(1.003,1.004)$ & 0.000 \\
\hline \multicolumn{6}{|l|}{ Occupation } \\
\hline Commercial services/civil servant & $7818(2.45)$ & 1.00 & & 1.00 & \\
\hline Agriculture & $249,093(78.14)$ & $1.42(1.36,1.49)$ & 0.000 & $1.25(1.19,1.31)$ & 0.000 \\
\hline Housekeeping ${ }^{\$}$ & $30,802(9.66)$ & $1.23(1.17,1.30)$ & 0.000 & $1.17(1.11,1.23)$ & 0.000 \\
\hline Education ${ }^{\Delta}$ & $10,679(3.35)$ & $0.80(0.75,0.85)$ & 0.000 & $0.84(0.79,0.90)$ & 0.000 \\
\hline Migrant worker & $2601(0.82)$ & $1.17(1.07,1.28)$ & 0.000 & $1.06(0.97,1.16)$ & 0.196 \\
\hline Healthcare & $1009(0.32)$ & $0.96(0.84,1.10)$ & 0.556 & $0.94(0.82,1.07)$ & 0.332 \\
\hline Hospitality & $612(0.19)$ & $0.80(0.67,0.94)$ & 0.009 & $0.82(0.69,0.97)$ & 0.022 \\
\hline Other & $16,178(5.07)$ & $1.06(1.01,1.12)$ & 0.027 & $0.98(0.93,1.04)$ & 0.508 \\
\hline \multicolumn{6}{|l|}{ Year } \\
\hline 2013 & $56,198(17.63)$ & 1.00 & & 1.00 & \\
\hline 2014 & $55,815(17.51)$ & $1.01(0.99,1.04)$ & 0.278 & $0.99(0.97,1.02)$ & 0.648 \\
\hline 2015 & $55,196(17.31)$ & $1.01(0.99,1.03)$ & 0.428 & $0.99(0.97,1.02)$ & 0.603 \\
\hline 2016 & $49,996(15.68)$ & $1.07(1.04,1.10)$ & 0.000 & $1.06(1.03,1.09)$ & 0.000 \\
\hline 2017 & $49,843(15.63)$ & $1.00(0.97,1.02)$ & 0.798 & $1.01(0.98,1.03)$ & 0.550 \\
\hline 2018 & $51,744(16.23)$ & $0.93(0.91,0.96)$ & 0.000 & $1.01(0.98,1.03)$ & 0.656 \\
\hline \multicolumn{6}{|l|}{ Residential address } \\
\hline Local & $310,343(97.35)$ & 1.00 & & 1.00 & \\
\hline Intra-provincial (within province) & $6215(1.95)$ & $1.23(1.17,1.30)$ & 0.000 & $1.48(1.41,1.56)$ & 0.000 \\
\hline Inter-provincial (between provinces) & $2182(0.68)$ & $0.86(0.79,0.94)$ & 0.001 & $1.06(0.98,1.16)$ & 0.153 \\
\hline Foreign nationality & $52(0.02)$ & $1.45(0.83,2.53)$ & 0.186 & $1.58(0.90,2.79)$ & 0.111 \\
\hline \multicolumn{6}{|l|}{ Patient enrolment classification } \\
\hline Consultation due to symptoms & $117,834(36.96)$ & 1.00 & & 1.00 & \\
\hline Referral & $103,261(32.39)$ & $0.66(0.65,0.67)$ & 0.000 & $0.65(0.63,0.66)$ & 0.000 \\
\hline Contact tracing & $93,183(29.23)$ & $0.73(0.72,0.74)$ & 0.000 & $0.74(0.72,0.75)$ & 0.000 \\
\hline Health check & $3179(1.00)$ & $0.18(0.17,0.20)$ & 0.000 & $0.20(0.18,0.22)$ & 0.000 \\
\hline Other & $1335(0.42)$ & $0.53(0.47,0.59)$ & 0.000 & $0.55(0.49,0.61)$ & 0.000 \\
\hline \multicolumn{6}{|l|}{ Diagnosis Institution } \\
\hline CDC & $278,707(88.15)$ & 1.00 & & 1.00 & \\
\hline Hospital & $33,104(10.47)$ & $0.92(0.90,0.95)$ & 0.000 & $0.93(0.91,0.96)$ & 0.000 \\
\hline TB dispensary & $4276(1.35)$ & $0.74(0.69,0.78)$ & 0.000 & $0.72(0.67,0.76)$ & 0.000 \\
\hline Other & $69(0.02)$ & $0.75(0.46,1.20)$ & 0.226 & $0.85(0.52,1.39)$ & 0.521 \\
\hline \multicolumn{6}{|l|}{ Severely III } \\
\hline No & $306,534(96.15)$ & 1.00 & & 1.00 & \\
\hline Yes & $12,258(3.85)$ & $1.31(1.00,1.02)$ & 0.000 & $1.35(1.31,1.41)$ & 0.000 \\
\hline
\end{tabular}


Table 4 (continued)

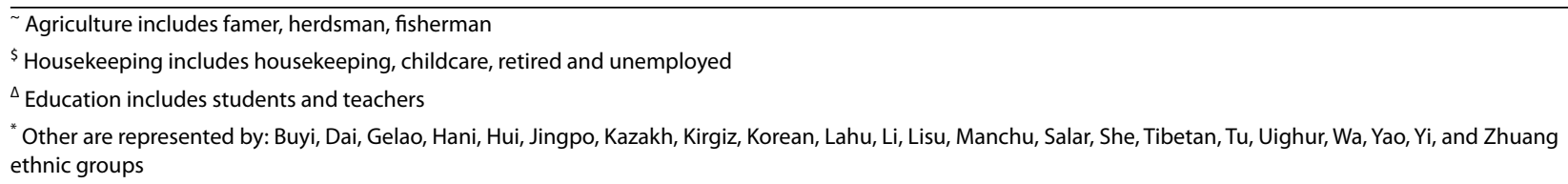

The negative binomial regression assessment of factors associated time from diagnosis to treatment commencement is detailed in the Additional file (Additional file 1: Table S4).

New patients represented $95.74 \%$ of the total study population and the results of the sensitivity analysis showed that there were no differences in treatment delays across the study variables between the two population groups (i.e., all TB patients vs. new patients only) (S1 Tables 5 and 6).

\section{Discussion}

Within Hunan Province, this study shows consistent and significant diagnosis delays for ethnic minority TB patients compared to the Han majority. However ethnic minority groups have lower odds of treatment delay relative to the Han majority.

Variables associated with TB diagnosis and treatment delay in previous studies include poverty, socio-economic disadvantage, knowledge, cultural beliefs, literacy, language, and distance and cultural barriers to health care provision [28-31]. The significant difference in the odds of TB diagnosis delay observed between Han majority and ethnic minority patients, and the differences observed between ethnic minority groups, may in part reflect socio-economic and cultural differences that have been reported to be associated with delay by previous studies. [28-31].

The ethnic minorities that inhabit Hunan occupy $28 \%$ of the province's land area [14], with approximately $96 \%$ occupying six cities and prefectures located within the 'Great Western Hunan' region [32], a region that is rural and less developed. In many rural and remote areas of China there is a lack of infrastructure and resources and a disparity in accessibility to services and facilities. [33, 34].

Although the disparity between urban and rural incomes in China is reducing, in 2019 the respective ratio was 2.59:1 in Hunan Province [35]. Disposable income is an important metric as TB patients face a myriad of direct (e.g., out of pocket medical expenses and health insurance exclusions/co-payments) and indirect costs (e.g., loss of income, cost of transport, food and accommodation) [7, 36-38]. Despite China successfully progressing its goal of universal public health insurance, catastrophic health expenditure (CHE) continues to be a significant confounder in effective TB diagnosis and treatment outcomes [9, 36, 39, 40]. 2016 figures estimate $15.11 \%$ of Chinese households experience CHE, with the rate 1.36 times higher in rural compared to urban households [41].

One of the most important confounders in the Chinese urban-rural income gap is education [42]. Improving ethnic minority educational attainment has been a high priority for China since 1949, prior to which it is estimated that up to $80 \%$ of its minority population were illiterate [43]. Despite the implementation of preferential policies however, lagging educational attainment continues to contribute to the Han-minority opportunity gap [44]. In addition to the differential between minority and majority populations, there is significant variation in educational attainment between minority groups [45].

The findings of this study show there are opportunities to reduce diagnosis delay within ethnic minority populations. The data supports integration of $\mathrm{TB}$ screening within routine health checks, a process that has been shown to be cost effective at improving case detection [46]. As evidenced by other studies, opportunities to improve patient seeking behaviour may relate to the socio-economic and cultural disadvantage experienced by ethnic minorities [13]. Health literacy is a key component of health seeking behaviour thereby reducing diagnosis delay. Population surveys in China show rural location and illiteracy to be significant risk factors in understanding TB and its symptoms [47]. Health seeking behaviour is also impacted by awareness of the NTP and distance to the nearest hospital [48]. Due to structural and economic constraints, patients in rural locations usually seek initial care within their own communities which often adds to the time delay in receiving a correct diagnosis [49].TB health seeking behaviour is also impacted by stigma of the disease, which in itself it impacted by social and cultural context $[49,50]$. Due to the significant diversity between and within different Chinese ethnic groups [44], detailed socioeconomic and cultural information is required to inform appropriate interventions.

When evaluating treatment delay, this study found all ethnic minority groups had lower odds of delay than the Han majority, with the finding significant in five of the seven ethnic minority groups. Further research is required to elucidate why the majority population is at greater risk of a treatment delay, and whether these findings are attributable to success of the NTP which 
Table 5 Univariable and multivariable regression of factors associated with 15 day treatment delay in TB patients registered in Hunan Province, 2013-2018

\begin{tabular}{|c|c|c|c|c|}
\hline & Univariable odds ratio $(95 \% \mathrm{Cl})$ & Univariable $p$ value & Multivariable odds ratio $(95 \% \mathrm{Cl})$ & $\begin{array}{l}\text { Multivariable } \\
p \text { value }\end{array}$ \\
\hline \multicolumn{5}{|l|}{ Ethnicity } \\
\hline Han & 1.00 & & 1.00 & \\
\hline Tujia & $0.75(0.71,0.78)$ & 0.000 & $0.92(0.88,0.96)$ & 0.000 \\
\hline Miao & $0.61(0.58,0.65)$ & 0.000 & $0.74(0.70,0.79)$ & 0.000 \\
\hline Dong & $0.77(0.72,0.84)$ & 0.000 & $0.87(0.81,0.95)$ & 0.001 \\
\hline Yao & $0.20(0.17,0.24)$ & 0.000 & $0.20(0.17,0.24)$ & 0.000 \\
\hline Bai & $0.67(0.54,0.84)$ & 0.000 & $0.83(0.65,1.05)$ & 0.126 \\
\hline Mongolian & $0.85(0.66,1.09)$ & 0.205 & $0.78(0.59,1.03)$ & 0.082 \\
\hline Other* & $0.69(0.52,0.93)$ & 0.014 & $0.70(0.51,0.97)$ & 0.030 \\
\hline \multicolumn{5}{|l|}{ Sex } \\
\hline Male & 1.00 & & 1.00 & \\
\hline Female & $1.11(1.09,1.13)$ & 0.000 & $1.07(1.05,1.09)$ & 0.000 \\
\hline Age & $0.998(0.998,0.999)$ & 0.000 & $1.001(1.0004,1.002)$ & 0.000 \\
\hline \multicolumn{5}{|l|}{ Occupation } \\
\hline Commercial services/civil servant & 1.00 & & 1.00 & \\
\hline Agriculture & $0.45(0.43,0.47)$ & 0.000 & $0.58(0.55,0.61)$ & 0.000 \\
\hline Housekeeping ${ }^{\$}$ & $0.85(0.81,0.90)$ & 0.000 & $0.81(0.76,0.86)$ & 0.000 \\
\hline Education $^{\Delta}$ & $0.64(0.60,0.68)$ & 0.000 & $0.67(0.63,0.72)$ & 0.000 \\
\hline Migrant worker & $0.41(0.37,0.46)$ & 0.000 & $0.64(0.57,0.72)$ & 0.000 \\
\hline Healthcare & $0.80(0.70,0.92)$ & 0.002 & $0.81(0.70,0.95)$ & 0.009 \\
\hline Hospitality & $0.87(0.73,1.03)$ & 0.115 & $0.91(0.75,1.10)$ & 0.320 \\
\hline Other & $0.68(0.64,0.72)$ & 0.000 & $0.82(0.77,0.87)$ & 0.000 \\
\hline \multicolumn{5}{|l|}{ Year } \\
\hline 2013 & 1.00 & & 1.00 & \\
\hline 2014 & $0.98(0.96,1.01)$ & 0.266 & $0.94(0.91,0.97)$ & 0.000 \\
\hline 2015 & $0.97(0.94,0.99)$ & 0.026 & $0.93(0.90,0.96)$ & 0.000 \\
\hline 2016 & $0.94(0.91,0.96)$ & 0.000 & $0.89(0.87,0.93)$ & 0.000 \\
\hline 2017 & $1.06(1.03,1.09)$ & 0.000 & $0.98(0.95,1.02)$ & 0.309 \\
\hline 2018 & $1.17(1.14,1.20)$ & 0.000 & $1.08(1.05,1.12)$ & 0.000 \\
\hline \multicolumn{5}{|l|}{ Residential address } \\
\hline Local & 1.00 & & 1.00 & \\
\hline Intra-provincial & $1.74(1.65,1.84)$ & 0.000 & $0.97(0.91,1.02)$ & 0.233 \\
\hline Inter-provincial & $1.74(1.60,1.90)$ & 0.000 & $1.14(1.04,1.26)$ & 0.008 \\
\hline Foreign nationality & $0.75(0.38,1.50)$ & 0.418 & $1.18(0.56,2.51)$ & 0.660 \\
\hline \multicolumn{5}{|l|}{ Patient enrolment } \\
\hline Consult-symptoms & 1.00 & & 1.00 & \\
\hline Referral & $3.62(3.53,3.72)$ & 0.000 & $3.62(3.52,3.72)$ & 0.000 \\
\hline Contact tracing & $14.44(14.07,14.83)$ & 0.000 & $14.45(14.06,14.84)$ & 0.000 \\
\hline Health check & $1.15(1.00,1.31)$ & 0.042 & $1.10(0.96,1.25)$ & 0.181 \\
\hline Other & $8.38(7.49,9.39)$ & 0.000 & $7.78(6.94,8.72)$ & 0.000 \\
\hline \multicolumn{5}{|l|}{ Diagnosis institution } \\
\hline CDC & 1.00 & & 1.00 & \\
\hline Hospital & $0.74(0.72,0.76)$ & 0.000 & $0.72(0.69,0.74)$ & 0.000 \\
\hline TB dispensary & $1.40(1.31,1.49)$ & 0.000 & $3.32(3.09,3.56)$ & 0.000 \\
\hline Other & $0.64(0.34,1.19)$ & 0.156 & $0.78(0.39,1.55)$ & 0.477 \\
\hline \multicolumn{5}{|l|}{ Severely III } \\
\hline No & 1.00 & & 1.00 & \\
\hline Yes & $0.86(0.83,0.90)$ & 0.000 & $0.73(0.69,0.76)$ & 0.000 \\
\hline
\end{tabular}


Table 5 (continued)

\footnotetext{
Agriculture includes famer, herdsman, fisherman

\$ Housekeeping includes housekeeping, childcare, retired and unemployed

${ }^{\Delta}$ Education includes students and teachers

* Other are represented by: Buyi, Dai, Gelao, Hani, Hui, Jingpo, Kazakh, Kirgiz, Korean, Lahu, Li, Lisu, Manchu, Salar, She, Tibetan, Tu, Uighur, Wa, Yao, Yi, and Zhuang ethnic groups
}

prioritizes vulnerable population groups $[9,10]$. Another possible explanation is that due to diagnosis delay in ethnic minorities, an increase in disease progression may lead to prioritization of treatment, however further research is required to test this hypothesis.

A significant strength of this study is the large, detailed data set on a well described cohort of patients. However, a lack of information on variables that may relate to the underlying causes of delay e.g., income and level of education, is a limitation of this study. The study is reliant upon the patient for the date of symptom onset from which diagnosis and treatment delay are calculated. Recall bias on the date of symptom onset therefore has the potential to impact subsequent findings. Only patients presenting for treatment at designated institutions are included in the analysis and so the data may not be representative of the variables across all TB patients within the province.

\section{Conclusions}

Reducing the time between TB onset and treatment is important in reducing morbidity and mortality and preventing further disease transmission This study shows ethnic minority groups experience significant TB diagnosis delay compared to the Han majority. Ethnicity is a complex variable that is often associated with a multitude of socio-economic disparities. These disparities are likely to be the underlying root cause of TB delay differentials observed between and within different population groups, which highlights the need for further research. It is also recommended that further studies evaluate the impact of ethnicity on TB treatment outcomes, as treatment outcomes are also key to effective TB control.

\footnotetext{
Abbreviations

AOR: Adjusted odds ratio; CDC: Centre for disease control and prevention; CHE: Catastrophic health expenditure; Cl: Confidence interval; COVID-19: Coronavirus disease 2019; DOTS: Directly observed treatment short-course; HIV: Human immunodeficiency virus; IQR: Interquartile range; IQR: Multi-drug resistant; NTP: National Tuberculosis Control Programme; $p$-value: Probability value; SD: Standard deviation; TB: Tuberculosis; TBCIHP: TB Control Institute of Hunan Province; VIF: Variance inflation factor; WHO: World Health Organization.
}

\section{Supplementary Information}

The online version contains supplementary material available at https://doi. org/10.1186/s12879-022-07072-4.
Additional file 1: Fig S1. Median time to diagnosis by ethnicity for TB patients registered in Hunan Province 2013-2018. Table S1. Sensitivity Analysis: Univariable and multivariable regression of factors associated with 14 day diagnosis delay in TB patients registered in Hunan Province, 2013-2018. Table S2. Univariable and multivariable negative binomial regression assessment of factors associated with time to diagnosis in TB patients registered in Hunan Province, 2013-2018. Table S3. Sensitivity Analysis: Univariable and multivariable regression assessment of factors associated with 1 day treatment delay in TB patients registered in Hunan Province, 2013-2018. Table S4. Univariable and multivariable negative binomial regression assessment of factors associated with time from diagnosis to treatment commencement in TB patients registered in Hunan Province, 2013-2018. Table S5. Median time from diagnosis to treatment commencement for new TB patients registered in Hunan Province, 20132018, by demographic characteristics. Table S6. Univariable and multivariable regression of factors associated with $>15$ day treatment delay in new TB patients registered in Hunan Province, 2013-2018.

\section{Acknowledgements}

Not applicable.

\section{Authors' contributions}

$B G, K A A$, and ACAC conceived the study. ZX and LB collected the data. BG undertook the analysis and prepared the draft manuscript. ACAC and KAA advised on the data analysis and the development of the manuscript. ZX and $L B$ revised the drafted manuscript. All authors critically revised the manuscript for methodological and intellectual content and have read and approved the final manuscript. All authors read and approved the final manuscript.

\section{Funding}

We acknowledge the Australian Government's support via an Australian Government Research Training Program Scholarship (BG) and a National Health and Medical Research Council Emerging Leadership Investigator Grant (KAA). The funders had no role in study design, decision to publish, or preparation of the manuscript.

\section{Availability of data and materials}

The datasets analysed during the current study are available from the corresponding author on reasonable request.

\section{Declarations}

\section{Ethics approval and consent to participate}

Ethics approval was obtained from Curtin University Human Research Ethics Committee (protocol number HRE2019-0581), and Hunan Chest Hospital provided written permission to access the data. All methods were performed in accordance with the relevant guidelines and regulations. As this study used secondary data, the need to obtain informed consent was waived by the Curtin University Human Research Ethics Committee.

\section{Consent for publication}

Not applicable.

\section{Competing interests}

The authors declare that they have no competing interests.

\section{Author details}

${ }^{1}$ Faculty of Health Sciences, Curtin University, Western Australia, Kent St, Bentley, WA 6102, Australia. ${ }^{2}$ Telethon Kids Institute, Nedlands, WA, Australia. 
${ }^{3}$ Xiangya School of Public Health, Central South University, Changsha, China.
${ }^{4}$ TB Control Institute of Hunan Province, Changsha, China.

Received: 9 August 2021 Accepted: 5 January 2022

Published online: 26 January 2022

\section{References}

1. ChildFund Australia. TB and COVID-19: fighting infectious diseases in PNG [Internet]. 2021. https://www.childfund.org.au/stories/tb-and-covid-19fighting-infectious-disease-in-png/. Accessed 06 Apr 2021.

2. TB Alliance. TB is a Pandemic [Internet]. n.d. https://www.tballiance.org/ why-new-tb-drugs/global-pandemic. Accessed 25 Feb 2021.

3. World Health Organization. Tuberculosis- Key Facts [Internet]. 2020. https://www.who.int/news-room/fact-sheets/detail/tuberculosis. Accessed 22 Feb 2021.

4. Daniel TM. The history of tuberculosis. Respir Med. 2006;100(11):1862-70. https://doi.org/10.1016/j.rmed.2006.08.006.

5. World Health Organization. TB Country, Regional and Global Profiles [Internet]. 2020. https://worldhealthorg.shinyapps.io/tb_profiles/. Accessed 22 Feb 2021.

6. Wang L, Liu J, Chin DP. Progress in tuberculosis control and the evolving public-health system in China. Lancet. 2007;369(9562):691-6.

7. Fang $\mathrm{H}$, Eggleston $\mathrm{K}$, Hanson $\mathrm{K}$, Wu M. Enhancing financial protection under China's social health insurance to achieve universal health coverage. BMJ. 2019. https://doi.org/10.1136/bmj.l2378.

8. Wang Z, Jiang W, Liu Y, Zhang L, Zhu A, Tang S, et al. Transforming tuberculosis (TB) service delivery model in China: issues and challenges for health workforce. Hum Resour Health. 2019;17(1):1-10.

9. Xu B, Dong H, Zhao Q, Bogg L. DOTS in China-removing barriers or moving barriers? Health Policy Plan. 2006;21(5):365-72.

10. The World Bank. China: Tuberculosis Control Project [Internet]. 2010 https://www.worldbank.org/en/news/feature/2010/09/24/china-tuber culosis-control-project0. Accessed 19 Apr 2021.

11. World Health Organization. Early detection of tuberculosis: an overview of approaches, guidelines and tools. Geneva: World Health Organization; 2011.

12. World Health Organization. The End TB Strategy; 2014. https://www.who. int/tb/strategy/End_TB_Strategy.pdf?ua=1. Accessed 23 Feb 2021.

13. Li Y, Ehiri J, Tang S, Li D, Bian Y, Lin H, et al. Factors associated with patient, and diagnostic delays in Chinese TB patients: a systematic review and meta-analysis. BMC Med. 2013;11(1):1-15.

14. Government of Hunan Province. The Official Website of the People's Government of Hunan Province [Internet]. 2021. http://www.enghunan. gov.cn/hneng/index.html. Accessed 22 Feb 2021.

15. World Health Organization. Tuberculosis in China [Internet]. n.d. https:// www.who.int/china/health-topics/tuberculosis. Accessed 23 Feb 2021.

16. Zhao L-L, Chen Y, Chen Z-N, Liu H-C, Hu P-L, Sun Q, et al. Prevalence and molecular characteristics of drug-resistant Mycobacterium tuberculosis in Hunan China. Antimicrob Agents Chemother. 2014;58(6):3475-80.

17. Zhang Y, Liu M, Wu SS, Jiang H, Zhang J, Wang S, et al. Spatial distribution of tuberculosis and its association with meteorological factors in mainland China. BMC Infect Dis. 2019;19(1):1-7.

18. Hunan Chest Hospital. Hunan Insititute for Tuberculosis Control, Hunan Chest Hospital [lnternet]. n.d. http://www.hnstb.com/. Accessed 23 Feb 2021.

19. World Health Organization. Diagnostic and treatment delay in tuberculosis. Geneva: World Health Organization; 2006.

20. World Health Organization. TB/HIV: a clinical manual. Geneva: World Health Organization; 2004.

21. Linzhu W. The identification of minorities in China. APLPJ. 2014;16:1.

22. Chimbatata NBW, Zhou C, Chimbatata CM, Mhango L, Diwan VK, Xu B. Barriers to prompt TB diagnosis - a comparative study between northern Malawi and eastern rural China. Trans R Soc Trop Med Hyg. 2017;111(11):504-11. https://doi.org/10.1093/trstmh/try002.

23. Abdu M, Balchut A, Girma E, Mebratu W. Patient delay in initiating tuberculosis treatment and associated factors in Oromia Special Zone Amhara Region. Pulm Med. 2020. https://doi.org/10.1155/2020/6726798.
24. Gebeyehu E, Azage M, Abeje G. Factors associated with patient's delay in tuberculosis treatment in Bahir Dar City administration, Northwest Ethiopia. BioMed Res Int. 2014. https://doi.org/10.1155/2014/701429.

25 Adenager GS, Alemseged F, Asefa H, Gebremedhin AT. Factors associated with treatment delay among pulmonary tuberculosis patients in public and private health facilities in Addis Ababa, Ethiopia. Tuberc Res Treat. 2017. https://doi.org/10.1155/2017/5120841.

26. Owolabi OA, Jallow AO, Jallow M, Sowe G, Jallow R, Genekah MD, et al. Delay in the diagnosis of pulmonary tuberculosis in The Gambia, West Africa: a cross-sectional study. Int J Infect Dis. 2020;101:102-6.

27. Fuge TG, Bawore SG, Solomon DW, Hegana TY. Patient delay in seeking tuberculosis diagnosis and associated factors in Hadiya Zone, Southern Ethiopia. BMC Res Notes. 2018;11(1):1-6.

28. Ngamvithayapong J, Yanai H, Winkvist A, Diwan V. Health seeking behaviour and diagnosis for pulmonary tuberculosis in an HIV-epidemic mountainous area of Thailand. Int J Tuberc Lung Dis. 2001;5(11):1013-20.

29 Hayward S, Harding RM, McShane H, Tanner R. Factors influencing the higher incidence of tuberculosis among migrants and ethnic minorities in the UK. F1000Res. 2018;7:461.

30 Roberts DJ, Mannes T, Verlander NQ, Anderson C. Factors associated with delay in treatment initiation for pulmonary tuberculosis. ERJ Open Res. 2020. https://doi.org/10.1183/23120541.00161-2019.

31. Huong NT, Vree M, Duong BD, Khanh VT, Loan VT, Borgdorff MW, et al. Delays in the diagnosis and treatment of tuberculosis patients in Vietnam: a cross-sectional study. BMC Public Health. 2007;7(1):1-8.

32. Hunan Provincial Bureau of Statistics. Hunan Overview, Population and Nationality [Internet]. 2021. https://www.hunan.gov.cn/hnszf/jxxx/ hngk/rkmz/rkmz.html. Accessed 01 Jul 2021.

33. Wang X, Pan J. Assessing the disparity in spatial access to hospital care in ethnic minority region in Sichuan Province, China. BMC Health Serv Res. 2016;16(1):1-11.

34. Cao H. Urban-rural income disparity and urbanization: what is the role of spatial distribution of ethnic groups? A case study of Xinjiang Uyghur Autonomous Region in western China. Reg Stud. 2010;44(8):965-82.

35. Hunan Government. Statistical communique on Hunan's econmonic and social development 2019 [Internet]. 2020 http://www.enghunan.gov.cn/ hneng/Business/EconomyCommunique/202004/t20200423_11969012. html. Accessed 01 Jul 2021.

36. $\mathrm{Ng} \mathrm{A}$, Dyckerhoff C, Then F. Private health insurance in China: finding the winning formula. Health Int. 2012;12:75-82.

37. Li F, Wu Y, Yuan Q, Zou K, Yang M, Chen D. Do health insurances reduce catastrophic health expenditure in China? A systematic evidence synthesis. PLoS ONE. 2020;15(9):e0239461.

38. Viney K, Islam T, Hoa NB, Morishita F, Lönnroth K. The financial burden of tuberculosis for patients in the Western-Pacific region. Tropical medicine and infectious disease. 2019;4(2):94.

39. Chen $S$, Zhang $H$, Pan $Y$, Long $Q$, Xiang $L$, Yao $L$, et al. Are free anti-tuberculosis drugs enough? An empirical study from three cities in China. Infect Dis Poverty. 2015;4(1):1-10.

40. Pan H, Bele S, Feng Y, Qiu S, Lü J, Tang S, et al. Analysis of the economic burden of diagnosis and treatment of tuberculosis patients in rural China. Int J Tuberc Lung Dis. 2013;17(12):1575-80.

41. Zhao Y, Oldenburg B, Mahal A, Lin Y, Tang S, Liu X. Trends and socioeconomic disparities in catastrophic health expenditure and health impoverishment in China: 2010 to 2016. Tropical Med Int Health. 2020;25(2):236-47.

42 Sicular T, Ximing Y, Gustafsson B, Li S. The urban-rural income gap and income inequality in China. In: Wan G, editor. Understanding inequality and poverty in China. London: Springer; 2008. p. 30-71.

43. Yang R, Wu M. Education for ethnic minorities in China: a policy critique. SA-eDUC Journal. 2009:6(2):117-31.

44. Hannum E, Wang M. China: a case study in rapid poverty reduction. In: Hall G, Patrinos H, editors. Indigenous peoples, poverty, and development. Cambridge: Cambridge University Press; 2012.

45. Bahry S. Minority education in China. In: Qiang Z, editor. Education in China: Educational history, models and initiatives. Berkshire Publishing Group, 2013. p. 234-241. 
46. Zhang X, Li S, Li H, Li G, Guo X, Wang Y, et al. Integrating tuberculosis screening into annual health examinations for the rural elderly improves case detection. Int J Tuberc Lung Dis. 2015;19(7):787-91.

47. Chen X, Wang W, Wang X, Chai C, Liu K, Peng Y, et al. Public awareness of tuberculosis in Southeast China: a population-based study. Int J Environ Res Public Health. 2019;16(21):4290.

48. Tong Y, Guan X, Hou S, Cai L, Huang Y, Wang L, et al. Determinants of health care-seeking delay among tuberculosis patients in rural area of central China. Int J Environ Res Public Health. 2018;15(9):1998.

49. Zhang T, Liu X, Bromley H, Tang S. Perceptions of tuberculosis and health seeking behaviour in rural Inner Mongolia. China Health policy. 2007;81(2-3):155-65.

50. Chang S, Cataldo J. A systematic review of global cultural variations in knowledge, attitudes and health responses to tuberculosis stigma. Int J Tuberc Lung Dis. 2014;18(2):168-73.

\section{Publisher's Note}

Springer Nature remains neutral with regard to jurisdictional claims in published maps and institutional affiliations.

- fast, convenient online submission

- thorough peer review by experienced researchers in your field

- rapid publication on acceptance

- support for research data, including large and complex data types

- gold Open Access which fosters wider collaboration and increased citations

- maximum visibility for your research: over 100M website views per year

At BMC, research is always in progress.

Learn more biomedcentral.com/submissions 